\title{
Suitability of the Binaural Interaction Component for Interaural Electrode Pairing of Bilateral Cochlear Implants
}

\author{
Hongmei Hu, Birger Kollmeier and Mathias Dietz
}

\begin{abstract}
Although bilateral cochlear implants (BiCIs) have succeeded in improving the spatial hearing performance of bilateral CI users, the overall performance is still not comparable with normal hearing listeners. Limited success can be partially caused by an interaural mismatch of the place-of-stimulation in each cochlea. Pairing matched interaural CI electrodes and stimulating them with the same frequency band is expected to facilitate binaural functions such as binaural fusion, localization, or spatial release from masking. It has been shown in animal experiments that the magnitude of the binaural interaction component (BIC) derived from the wave-eV decreases for increasing interaural place of stimulation mismatch. This motivated the investigation of the suitability of an electroencephalography-based objective electrode-frequency fitting procedure based on the BIC for BiCI users. A 61 channel monaural and binaural electrically evoked auditory brainstem response (eABR) recording was performed in 7 MED-EL BiCI subjects so far. These BiCI subjects were directly stimulated at $60 \%$ dynamic range with 19.9 pulses per second via a research platform provided by the University of Innsbruck (RIB II). The BIC was derived for several interaural electrode pairs by subtracting the response from binaural stimulation from their summed monaural responses. The BIC based pairing results are compared with two psychoacoustic pairing methods: interaural pulse time difference sensitivity and interaural pitch matching. The results for all three methods analyzed as a function of probe electrode allow for determining a matched pair in more than half of the subjects, with a typical accuracy of \pm 1 electrode. This includes evidence for statistically significant tuning of the BIC as a function of probe electrode in human subjects. However, results across the three conditions were sometimes not consistent. These discrepancies will be discussed in the light of pitch plasticity versus less plastic brainstem processing.
\end{abstract}

Keywords Binaural interaction component - Electrically evoked auditory brainstem response $\cdot$ Bilateral cochlear implant $\cdot$ Pitch matching $\cdot$ Interaural pulse time difference $\cdot$ Interaural electrode pairing

H. Hu $(\bowtie) \cdot$ B. Kollmeier · M. Dietz

Medizinische Physik, Universität Oldenburg, Cluster of Excellence "Hearing4all",

26111 Oldenburg, Germany

e-mail: hongmei.hu@uni-oldenburg.de

(C) The Author(s) 2016

P. van Dijk et al. (eds.), Physiology, Psychoacoustics and Cognition in Normal

and Impaired Hearing, Advances in Experimental Medicine and Biology 894,

DOI 10.1007/978-3-319-25474-6_7 


\section{Introduction}

Bilateral cochlear implantation seeks to restore the advantages of binaural hearing to the profound deaf by providing binaural cues that are important for binaural perception, such as binaural fusion, sound localization and better detection of signals in noise. Most bilateral cochlear implant (BiCI) users have shown improvements compared to their ability when only one $\mathrm{CI}$ was used. However, compared to normal hearing $(\mathrm{NH})$ individuals, the average performance of BiCI users is still worse and has a large variability in performance amongst them (Majdak et al. 2011; Litovsky et al. 2012; Goupell et al. 2013; Kan et al. 2013). One likely reason for the worse performance of $\mathrm{BiCI}$ users is the interaural electrodes mismatch between two CIs because of different surgery insertion depth or different implant length. Since the inputs to the NH binaural system from the two ears can be assumed to be well matched and binaural brainstem neurons are comparing only by interaurally place matched inputs, it is very important to determine interaural electrode pairs for frequency matching the electrode arrays in the two ears to compensate for any differences in the implanted cochleae. This interaural electrode pairing (IEP) is expected to become even more relevant in the future, to better exploit recent time information preserving coding strategies and with the advent of truly binaural cochlear implants, which will preserve, enhance, and/or optimize interaural cues.

It has been suggested that physiological measures of binaural interactions (e.g., evoked potentials) will likely be required to accomplish best-matched interaural electrode pairs (Pelizzone et al. 1990). More recently, the binaural interaction component (BIC) of the electrically evoked auditory brainstem responses (eABRs) have been obtained in both animals (Smith and Delgutte 2007) and humans (He et al. 2010; Gordon et al. 2012). Smith and Delgutte (2007) proposed a potential way by using evoked potentials to match interaural electrode pairs for bilaterally implanted cats. Their study shows that the interaural electrode pairings that produced the best aligned IC activation patterns were also those that yielded the maximum BIC amplitude. More recently, He et al. (2010) observed some evidence of a BIC/electrodeoffset interaction at low current levels. In another follow up study, they used the same electroencephalography (EEG) procedure to examine whether the BIC amplitude evoked from different electrode pairings correlated with an interaural pitch comparison task (He et al. 2012). Their results show that there is no significant correlation between results of BIC measures and interaural pitch comparisons on either the individual or group levels. Gordon et al. (2012) demonstrated that binaural processing in the brainstem of children using bilateral $\mathrm{CI}$ occurs regardless of bilateral or unilateral deafness; it is disrupted by a large but not by a small mismatch in place of stimulation. All these studies suggest that BIC could be a potentially approaches for electrode pairing, especially for pediatrics, however, it is not accurate enough in all existing studies of human subjects.

In order to tackle the issue of accuracy, a 61-channel monaural and binaural eABR recording setup was developed together with a multi-step offline post-pro- 
cessing strategy specifically for eABR signals. Further, to address the question of method validity the BIC based pairing results are compared with two psychoacoustic pairing methods: interaural pulse time difference sensitivity and interaural pairwise pitch comparison.

\section{Methods}

Seven BiCIs (three male and four female; mean age $=53$ year) participated in this study. All of them were post-lingual at their onset of bilateral severe-to-profound sensorineural hearing loss and were using MED-EL implant systems. The voluntary informed written consent was obtained with the approval of the Ethics Committee of the University of Oldenburg. The intra-cochlear electrodes of the MED-EL CI are numbered from 1 to 12 in an apical to basal direction. Here the electrode on the right or the left implant is named Rx or Lx. For example, L4 refers to the 4th electrode on the left implant.

A research platform was developed for psychoacoustic testing and eABR recording (Hu et al. 2014). The electrical stimuli were presented via a research interface box (RIB II, manufactured at University of Innsbruck, Austria) and a National Instruments I/O card. The monaural or bilaterally synchronized electrical pulses were applied to the CI, bypassing the behind-the-ear unit.

The experiment consisted of three psychophysical pretests, accompanied by three IEP methods. Pretest 1 was to determine the participant's maximum comfortable level (MCL) and the hearing threshold (HL) of the reference electrode (For more details about the stimuli, please refer to the last paragraph of section 2). From these two values the dynamic range (DR) was obtained. In the second pretest the interaural loudness-balanced level for each electrode pair was determined. The presentation level of the reference CI electrode was fixed at $60 \%$ of DR, while the presentation level of the contralateral probe CI electrode was adapted according to the participant's response. The stimulation levels judged by the participant to be equally loud in both sides were saved and used in the pairwise pitch comparison and EEG recording procedures. In the interaural pairwise pitch comparison task interaural electrode pairs were stimulated sequentially. The participants were asked to indicate in which interval the higher pitch was perceived. This experiment was repeated 50 times per electrode pair. As a third pre-test, a lateralization task was performed on each electrode pair to ensure that a single, fused auditory image was perceived in the center of the head (Kan et al. 2013). The current level and electrode pairs that allowed for a fused and central image were again stored and employed for the second IEP method: IPTD discrimination. Two-interval trials, randomly located with left-leading (IPTD $=-\mathrm{T} / 2$ ) and right-leading stimuli (IPTD $=\mathrm{T} / 2$ ), were presented for the electrode pair using a constant stimulus procedure, for a fixed IPTD $=\mathrm{T}$. The participant was required to indicate whether the stimulus in the second interval was perceived either to the left or the right of the first interval. 
In the EEG measurement session, eABRs were differentially recorded from $\mathrm{Ag} /$ $\mathrm{AgCl}$ electrodes of a customized equidistant 63-channel braincap (Easycap) with an electrode at FPz serving as the ground, and the midline cephalic location $(\mathrm{Cz})$ as the physical reference (Hu et al. 2015). The 63 scalp electrodes were connected to the SynAmps RT amplifier system (Neuroscan). Channel 49 (sub-Inion) and channel 59 (Inion) were primary interest in this study. Electrode impedances were kept below $10 \mathrm{~K} \Omega$. The sweeps were filtered by an analog antialiasing-lowpass with a corner frequency of $8 \mathrm{kHz}$, digitized with $20 \mathrm{kHz}$ sampling rate via a 24 bit A/D convertor. The artifact rejection was turned off during the recording, since filtering, artifact analysis and averaging were done offline. 2100-3000 single sweeps for all electrode conditions were recorded in random order on a sweep-by-sweep basis. During the recording, the participants seated in a recliner and watched silent subtitled movies within an electrically shielded sound-attenuating booth.

The stimulus was a train of charge-balanced biphasic pulses presented at a rate of 19.9 pulses per second (pps) (He et al. 2010), with 50 or $60 \mu$ s phase duration, and $2.1 \mu$ s interphase gap presented repeatedly via monopolar stimulation mode. A phase duration of $60 \mu$ s was used only when the subject could not reach the MCL with $50 \mu \mathrm{s}$. In psychophysical sessions 1-4, a 10-pulse chain about $500 \mathrm{~ms}$ time duration was used. In the IPTD test, each interval was a $500 \mathrm{~ms}$ stimulus and there was 300 ms pause between the two intervals. For the EEG recoding, a continuous electrical pulse train was presented with a $5 \mathrm{~ms}$ trigger sent $25 \mathrm{~ms}$ before each the CI stimulation onset.

\section{Results}

The BIC is defined as the difference between the eABR with binaural stimulation (B) and the sum of the eABRs obtained with monaural stimulation $(\mathrm{L}+\mathrm{R})$ : $\mathrm{BIC}=$ $\mathrm{B}-(\mathrm{L}+\mathrm{R})$. For both eABR and BIC, the latency was defined as the time position of the respective peaks. The amplitude was defined as the difference between the positive peak and the following trough amplitude.

Figure 1a shows the morphology of the eABR and the BIC of subject S5 with reference electrode L4 and probe electrode R1, where the electric artifact was already removed (Hu et al. 2015). In general, wave eI was not observed due to the stimulation pulse artifact from the implant. The amplitude of wave $\mathrm{eV}$ was larger than an acoustically evoked ABR amplitude, and latency which occurs approximately $3.6 \mathrm{~ms}$ after the onset of the stimulus is shorter because the electrical stimulus directly activates the neural pathway (Starr and Brackmann 1979; Pelizzone et al. 1989). Figure $1 \mathrm{~b}$ shows the results of the BIC across 12 electrode pairs (R1-R12), for S5 with reference electrode of L4. The latency of the BIC is about $4 \mathrm{~ms}$, and the BIC amplitude is increasing first and then decreasing after electrode 6 (R6). Figure 1c shows the IEP results based on the best IPTD performance and the pitch comparison. The open circles on the dash-dotted line indicate the per- 
a

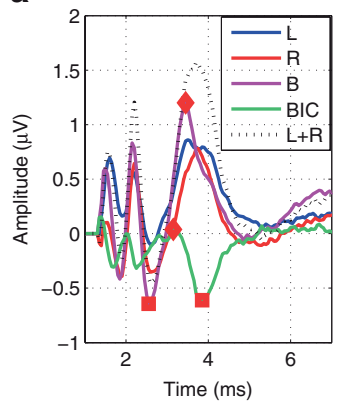

b

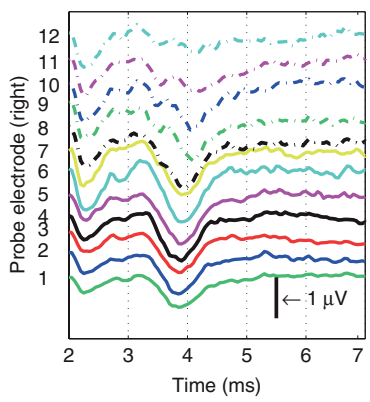

C

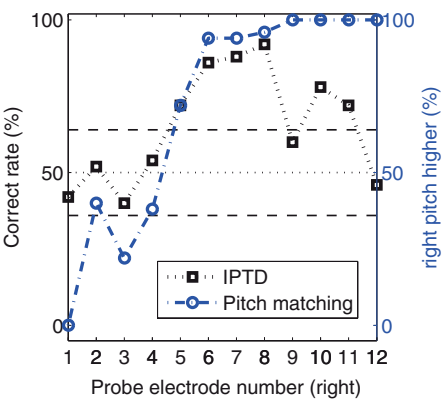

Fig. 1 EABRs and the BICs of subject S5 and the reference electrode is L4: a EABRs of left CI stimulated only, right CI stimulated only, both CI stimulated simultaneously, L + R and the BIC. The probe electrode is R1. The $\mathrm{y}$-axis is their amplitude values in $\mu \mathrm{V}$. The electric artifact was lined out, wave $\mathrm{eV}$ and $\mathrm{BIC}$ are visible at approximately 3.6 and $4.1 \mathrm{~ms}$, respectively. b The results of the BIC across 12 electrode pairs. $\mathbf{c}$ The IEP results based on the best IPTD performance and pitch comparison. The $\mathrm{x}$-axis is the number of the probe electrodes in the right implant

centage of how often the probe electrode (right side) resulted in a higher pitch percept than the reference electrode (left side) in the pitch matching experiment. The squares on the dashed line are the correct rates of lateralization judgment in the IPTD experiment. The $\mathrm{x}$-axis indicates the probe electrode number in the right implant. The matched electrodes are R4.4 (pitch comparison), R6-R8 (best IPTD performance), and R6 (largest BIC amplitude). In contrast to the common assumption that pitch-matched pairs maximize sensitivity to binaural cues, for this subject the pairs with best IPTD sensitivity are 1.6-3.6 electrodes away from (interpolated) electrode identical pitch perception. The BIC based method indicates electrode R6 to be matched with L4, which is closer to the IPTD based match for this subject. For S5, there are three pairs that have a high IPTD sensitivity (R6 -R8). They are not significantly different from each other, rendering the assignment of a unique matched pair difficult. This is consistent with the previous finds that ITD JNDs do not change much until there is a 3-6 mm mismatch (van Hoesel and Clark 1997). Note that the pitch matched electrode pair does not even yield a significantly above chance IPTD sensitivity.

The results from the other methods and of the other subjects are not shown because of the space limitations. The general trends were: (1) Pairwise pitch comparison results highly depend on the participant's clinical frequency-place fitting map. The matched pair indicates a similar frequency range in the map, except for one subject which was implanted only 9 months before the experiment. (2) Both BIC and IPTD based pairing results indicate a large 2-3 electrode mismatch for some subjects. There is clear correlation between the mismatches indicated by these two methods. (3) In line with Poon et al. (2009) there is no correlation between the pitch matched pairs and the IPTD matched pair and in line with He et al. (2012) there is no correlation between the pitch matched pairs and the BIC matched pair. 


\section{Discussion}

With the increasing number of bilateral CI users and new product development towards binaural CIs, it becomes increasingly important to find ways of matching the electrode arrays in the two ears to compensate for the differences between the two implanted cochleae because of insertion depth, electrode lengths, or neural survival. Current clinical cochlear implant fitting strategies generally treat each implant monaurally and allocate a generic frequency range to each electrode based on the electrode number often without concern about the actual position and the mismatching between the two implants. In a very few positive exceptions interaural pitch comparison techniques are employed. This study investigated the suitability of three IEP methods which have previously been tested in research studies but mostly only one method in isolation. In general, clear monaural and binaural eABRs and BICs were obtained in most of the subjects. All but one subject showed nicely tuned pitch matching, IPTD sensitivity, and BIC as a function of electrode number. However, the pairing results were not always consistent across methods. The IPTD paring results of some subjects are spanned across several electrodes, which is consistent with the previous finds that ITD JNDs do not changed much until there is a $3-6 \mathrm{~mm}$ mismatch compared to the best matched place condition (van Hoesel and Clark 1997).

Interaural pitch comparison shows some predictive value in selecting interaural electrode pairs, but it may not fully compensate for any underlying differences between the two implanted cochleae. In line with previous studies (van Hoesel and Clark 1997; Long et al. 2003; Poon et al. 2009) the pitch matched pair does not guarantee best IPTD sensitivity. All the CI subjects in this study have had at least 9 months bilateral $\mathrm{CI}$ experience prior to the experiment so acclimatization to the electrode-frequency-map or brain plasticity may explain the good pitch tuning curve. There is no correlation between the BIC amplitudes and results of interaural pitch matching as well, which is consistent with (He et al. 2012).

For the interpretation of the BIC, the straightest assumption is that if the reference electrode and the probe electrode are cochlear place matched, they stimulate similarly "tuned" auditory-nerve fibers that eventually result in binaural interaction in the brain stem and thus in a larger BIC than the other pairs. However, in case of a different neural survival across the ears or within one ear, also non-place matched electrodes may result in the maximum BIC. Further studies with more reference electrodes are necessary to test this assumption. This may also partially explain the similar ITD sensitivity of the neighboring electrodes around the best matched electrode.

In summary, all three methods can obtain reasonable interaural electrode pairing results. There is substantial variability in all the measurements both within and between individual CI users. There are pros and cons for all the three methods and there is no gold standard to judge which one is better. The acclimatization to the frequency to place map of the patient's CI seems unignorably in the pitch perception. Pitch matching data may be 'misleadingly good' because of acclimatization or brain 
plasticity which does not affect the IPTD and the BIC based matching. Knowledge about the location of the implant inside the cochlea maybe helpful in judging the pairing results. Longitudinal measurements are necessary in the future to investigate the plasticity of the binaural perception in the $\mathrm{BiCI}$ users. The $\mathrm{BIC}$ is a promising candidate for electrode pairing of BiCI subjects, especially for pediatric fitting.

Acknowledgments This work was funded by European Union under the Advancing Binaural Cochlear Implant Technology (ABCIT) grant agreement (No. 304912). We thank our ABCIT project partners at the UCL Ear Institute David McAlpine and Torsten Marquardt for inspirational discussions when planning this study. The authors are grateful to Stefan Strahl (MED-EL) for RIB II support and Stephan Ewert and Tom Campbell for their help during method development. We thank Karsten Plotz, Katrin Bomke (Klinik für Phoniatrie und Pädaudiologie, Ev. Krankenhaus Oldenburg), and Stefan Dazert (CI-Zentrum Ruhrgebiet, Universitätsklinik Bochum) for recruiting CI participants. The authors also thank all CI participants for joining the study and for giving us valuable feedback.

Open Access This chapter is distributed under the terms of the Creative Commons AttributionNoncommercial 2.5 License (http://creativecommons.org/licenses/by-nc/2.5/) which permits any noncommercial use, distribution, and reproduction in any medium, provided the original author(s) and source are credited.

The images or other third party material in this chapter are included in the work's Creative Commons license, unless indicated otherwise in the credit line; if such material is not included in the work's Creative Commons license and the respective action is not permitted by statutory regulation, users will need to obtain permission from the license holder to duplicate, adapt or reproduce the material.

\section{References}

Gordon KA, Salloum C, Toor GS, van Hoesel R, Papsin BC (2012) Binaural interactions develop in the auditory brainstem of children who are deaf: effects of place and level of bilateral electrical stimulation. J Neurosci 32(12):4212-4223

Goupell MJ, Stoelb C, Kan A, Litovsky RY (2013) Effect of mismatched place-of-stimulation on the salience of binaural cues in conditions that simulate bilateral cochlear-implant listening. J Acoust Soc Am 133(4):2272-2287

He S, Brown CJ, Abbas PJ (2010) Effects of stimulation level and electrode pairing on the binaural interaction component of the electrically evoked auditory brain stem response. Ear Hear 31(4):457-470

He S, Brown CJ, Abbas PJ (2012) Preliminary results of the relationship between the binaural interaction component of the electrically evoked auditory brainstem response and interaural pitch comparisons in bilateral cochlear implant recipients. Ear Hear 33(1):57-68

Hu H, Ewert S, Campbell T, Kollmeier B, Dietz M (2014). An interaural electrode pairing clinical research system for bilateral cochlear implants. Paper presented at the the 2nd IEEE China Summit and International Conference on Signal and Information Processing (ChinaSIP' 14), Xi'an, China

Hu H, Kollmeier B, Dietz M (2015 Reduction of stimulation coherent artifacts in electrically evoked auditory brainstem responses. Biomed Signal Process Control xx(in Press):xx-xx

Kan A, Stoelb C, Litovsky RY, Goupell MJ (2013) Effect of mismatched place-of-stimulation on binaural fusion and lateralization in bilateral cochlear-implant users. J Acoust Soc Am 134(4):2923-2936 
Litovsky RY, Goupell MJ, Godar S, Grieco-Calub T, Jones GL, Garadat SN, Agrawal S, Kan A, Todd A, Hess C, Misurelli S (2012) Studies on bilateral cochlear implants at the University of Wisconsin's Binaural Hearing and Speech Laboratory. J Am Acad Audiol 23(6):476-494

Long CJ, Eddington DK, Colburn HS, Rabinowitz WM (2003) Binaural sensitivity as a function of interaural electrode position with a bilateral cochlear implant user. J Acoust Soc Am 114(3):1565-1574

Majdak P, Goupell MJ, Laback B (2011) Two-dimensional localization of virtual sound sources in cochlear-implant listeners. Ear Hear 32(2):198-208

Pelizzone M, Kasper A, Montandon P (1989) Electrically evoked responses in Cochlear implant patients. Audiology 28(4):230-238

Pelizzone M, Kasper A, Montandon P (1990) Binaural interaction in a cochlear implant patient. Hear Res 48(3):287-290

Poon BB, Eddington DK, Noel V, Colburn HS (2009) Sensitivity to interaural time difference with bilateral cochlear implants: development over time and effect of interaural electrode spacing. J Acoust Soc Am 126(2):806-815

Smith ZM, Delgutte B (2007) Using evoked potentials to match interaural electrode pairs with bilateral cochlear implants. J Assoc Res Otolaryngol 8(1):134-151

Starr A, Brackmann DE (1979) Brain stem potentials evoked by electrical stimulation of the cochlea in human subjects. Ann Otol, Rhinol Laryngol 88(4 Pt 1):550-556

van Hoesel RJ, Clark GM (1997) Psychophysical studies with two binaural cochlear implant subjects. J Acoust Soc Am 102(1):495-507 